\section{Storage test on apple juice after ultrasound treatment}

\author{
Filomena Montemurro, ${ }^{1}$ Luca Fasolato, ${ }^{1}$ \\ Stefania Balzan, ${ }^{1}$ Roberta De Nardi, ${ }^{2}$ \\ Giorgio Marchesini, ${ }^{2}$ Barbara Cardazzo, ${ }^{1}$ \\ Enrico Novelli ${ }^{1}$
}

1 Dipartimento di Biomedicina Comparata e Alimentazione, Università degli Studi di Padova; 'Dipartimento di Medicina Animale, Produzioni e Salute, Università degli Studi di Padova, Legnaro (PD), Italy

\section{Abstract}

Apple juice, for its sensory and nutritional qualities, is consumed by people of all ages. Apples are an excellent source of several phenolic compounds and the presence of polyphenols is recognized for their health promoting antioxidant properties. Thermal pasteurization of fruit juices is the conventional method used for their preservation. Therefore, this constitutes the most extensively available methods for the inactivation of microorganisms in fruit juices but it causes side effects on their flavour and nutritional quality. Consumers tend to prefer recently extracted juices with fresh taste and minimal flavor or vitamin losses. To meet consumers' demand, among the novel technologies that involve non-thermal processes, power ultrasound have been investigated as an alternative to conventional heat treatments. Objective of this study was to evaluate the effectiveness of the use of ultrasound in an attempt to maintain the organoleptic characteristics typical of a natural apple juice. In particular, it was evaluated the action on the microflora residing and shelf life of the product through microbiological and sensory analyses. Juice treated with ultrasound highlighted a reduction of aerobic mesophilic counts and psychrophilic bacteria respectively about 3 and $5 \log \mathrm{CFU} / \mathrm{mL}$ and an enhanced yeast growth. The general opinion expressed by the panelist was in favour of the sonicated juice. This preliminary study showed that non-thermal methods such as power ultrasound technology may give new opportunities to develop fresh-like apple juice.

\section{Introduzione}

Il succo rappresenta il principale prodotto della trasformazione industriale delle mele (Pompei, 2005). Alimento ricco di antiossidanti, ferro e vitamina $\mathrm{C}$, povero di sodio e grasso è apprezzato e consumato da persone di tutte le età (Lee et al., 2003; Leontowicz et al., 2003). Secondo dati riportati da MARK UP, mensile de Il Sole24 ore, il consumo italiano medio pro capite di succhi di frutta è di circa $15 \mathrm{~L}$ annui. La grande tendenza per questi prodotti è il ritorno al naturale, con un forte accento ai prodotti funzionali e ai nuovissimi frullati (smoothies) che si consumano al posto della frutta fresca. Anche i classici succhi si rinnovano attraverso il minimo impiego della chimica, niente zuccheri aggiunti e assenza di additivi antiossidanti (Dalpozzo, 2010). Il succo di mela fresco, come altri succhi di frutta, è suscettibile al deperimento causato da batteri e dall'attività enzimatica che ne riducono la shelf life, attraverso la produzione di off-flavours, aumento della torbidità e produzione di gas. Le alterazioni che più spesso interessano il succo di mela sono causate principalmente da lieviti come Sacharomyces cerevisiae, ma anche batteri patogeni come Escherichia coli e Listeria monocytogenes sono stati identificati in questi prodotti (Guerrero-Beltrán e Barbosa-Cánovas, 2005). Grazie al suo pH acido, il succo di mela non è considerato un substrato idoneo per i patogeni alimentari, tuttavia la capacità di Escherichia coli 0157:H7 di resistere a valori bassi di $\mathrm{pH}$ ha determinato casi di malattie alimentari in seguito al consumo di succo di mela. Dal 1995 al 2005 sono stati denunciate al Centers for Disease Control and Prevention (CDC; Atlanta, GA, USA) 21 casi di malattia associati al consumo di succo e 10 di questi erano riconducibili all'ingestione di succo di mela (Vojdani et al., 2008). Attualmente il trattamento termico di pastorizzazione è il metodo convenzionale utilizzato per la conservazione del succo. Questo processo ha diversi effetti sulla qualità del prodotto che ne pregiudicano l'accettabilità generale, uno tra tutti l'imbrunimento conseguente all'ossidazione dei composti fenolici (Bump, 1989) e alle reazioni di Maillard. Tuttavia, i consumatori preferiscono succhi da consumare a breve distanza di tempo dalla produzione, che presentino un gusto fresco e una minima perdita dell'aroma tipico (Bignon, 1996). Per soddisfare queste esigenze negli ultimi anni sono stati studiati diversi metodi alternativi al trattamento termico quali le radiazioni ultraviolette (UV-C) (Donahue et al., 2004; Franz et al., 2009; Wright et al., 2000), i campi elettrici pulsati (Ayhan et al., 2002; Evrendilek et al., 1999) e gli ultrasuoni (Adekunte et al., 2010; D'Amico et al., 2006; Patil et al., 2009; SallehMark e Roberts, 2007). Tra questi la sonicazione, ovvero il trattamento con ultrasuoni (US), da sola 0 in combinazione con un blando trattamento termico (termosonicazione), si è dimostrata efficace nei confronti di batteri quali coliformi, Salmonella spp., Listeria monocyogenes, e altri microrganismi (Piyasena et al., 2003), capace di determinare abbattimenti della popolazione microbica fino
Correspondence: Filomena Montemurro, Dipartimento di Biomedicina Comparata e Alimentazione, Università degli Studi di Padova, Legnaro (PD), Italy.

Tel. +39.049 .8272965 - Fax: +39.049 .8272604 .

E-mail: filomena.montemurro@studenti.unipd.it

Key words: Apple juice, Power ultrasound, Shelf life, Sensory analysis.

Acknowledgments: the authors would like to thank Fondazione Cariverona.

Received for publication: 15 January 2013. Revision received: 30 September 2013

Accepted for publication: 30 September 2013.

This work is licensed under a Creative Commons Attribution 3.0 License (by-nc 3.0).

CC Copyright F. Montemurro et al., 2014

Licensee PAGEPress, Italy

Italian Journal of Food Safety 2014; 3:955

doi:10.4081/ijfs.2014.955

a $5 \log$ in tempi più brevi rispetto al solo trattamento termico (Bermùdez-Aguirre et al., 2009).

Gli high power ultrasounds, caratterizzati da frequenze comprese tra i 20 ed i $100 \mathrm{KHz}$, se utilizzati su matrici fluide provocano il fenomeno della cavitazione. La compressione e rarefazione originate dall'onda sonora portano alla formazione di microbolle che implodendo producono un'onda d'urto che si propaga nel fluido danneggiando le membrane cellulari dei microrganismi presenti (Chisti, 2003). Parallelamente a quest'azione battericida, gli US sembrano in grado di prolungare la shelf life degli alimenti trattati inibendo l'attività enzimatica endogena (Kelly e Zeece, 2009) e, nel caso del latte, sembrerebbero ridurre le dimensioni dei globuli di grasso rendendolo più omogeneo (Villamiel e de Jong, 2000) e più apprezzato dal consumatore.

Obiettivo del presente lavoro è stato quello di valutare l'efficacia dell'utilizzo degli ultrasuoni, come trattamento alternativo all'applicazione del calore nel tentativo di mantenere le caratteristiche organolettiche proprie di un succo di mela naturale. In particolare si è valutata l'azione sulle microflore residenti e la shelf life del prodotto. Quanto proposto costituisce una valutazione preliminare che si inserisce in un più ampio progetto di studio riguardante l'applicazione dei trattamenti di sonicazione su matrici alimentari fluide.

\section{Materiali e Metodi}

Al fine di valutare l'effetto complessivo dell'applicazione degli ultrasuoni sulle caratteri- 
stiche igieniche e sensoriali del succo di mela, si è proceduto con la spremitura di mele gialle Golden Delicious (Melinda D.0.P. 4021) utilizzando una centrifuga Ariete (Centrika metal). Le mele, in attesa della spremitura, sono state lavate, porzionate conservando la buccia e mantenute in acqua pulita, alla quale è stato aggiunto acido ascorbico (circa 0,05\%) (Pompei, 2005) e ghiaccio. Successivamente alla centrifugazione il succo è stato trattato con ultrasuoni utilizzando un sonicatore modello UP 400S (Hielscher USA, Inc., Ringwood, NJ, USA) con potenza, frequenza e lunghezza d'onda pari a $400 \mathrm{~W}, 24 \mathrm{kHz}$ e $120 \mathrm{~m}$ rispettivamente, su cui è stata montata una sonda da $22 \mathrm{~mm}$ di diametro. L'applicazione è stata effettuata in batch per immersione in un volume di succo da trattare pari a $500 \mathrm{~mL}$. Al fine di impedire il surriscaldamento del succo, il contenitore è stato mantenuto in bagno di ghiaccio durante il trattamento.

Lo studio ha considerato tre tesi: ) succo di mela trattato con ultrasuoni (S). Il trattamento effettuato è caratterizzato da un valore di ampiezza dell'onda (A) pari al 100\%, durata di sonicazione (D) $300 \mathrm{~s} / 100 \mathrm{~mL}$ e pulsazione (P) pari a 1 (quando il livello di pulsazione è 1 la sonicazione è detta in continuo); ii) succo di mela riscaldato applicando una cinetica di temperatura sovrapponibile a quanto rilevato durante il trattamento di sonicazione (T); iii) succo di mela non trattato (C).

Ogni campione, dopo il trattamento è stato portato rapidamente a temperatura di refrigerazione utilizzando un bagno di ghiaccio e mantenuto in regime di refrigerazione fino al momento dell'analisi. Ogni trattamento è stato effettuato in triplicato.

Al fine di valutare l'effetto degli ultrasuoni sulla flora microbica, sono stati effettuati 4 campionamenti in un intervallo di tempo di 21 gg (T0: giorno del trattamento; T7 : dopo 7 gg; T14: dopo 14gg; T21 dopo 21gg). Sono state allestite le opportune diluizioni con Maximum Recovery Diluent (MRD; 0xoid, Basingstoke, UK) ed effettuate le semine di ciascuna diluizione rispettivamente su Plate Count Agar (PCA; 0xoid) incubato a $30^{\circ} \mathrm{C}$ per $72 \mathrm{~h}$ per la determinazione dei mesofili e a $4^{\circ} \mathrm{C}$ per 10 giorni per gli psicrofili; su Oxytetracycline Glucose Agar (OGA; Biokar, Ho Chi Minh City, Vietnam) incubato a $25^{\circ} \mathrm{C}$ per $3 \mathrm{gg}$ per i lieviti e 5 gg per le muffe; su MRS Agar With Tween 80 (MRSA) per $48 \mathrm{~h}$ a $30^{\circ} \mathrm{C}$ per i lattobacilli mesofili e 24 h a $22^{\circ} \mathrm{C}$ per gli psicrofili e su Coli ID (bioMérieux, Marcy l'Etoile, Francia) incubato a $37^{\circ} \mathrm{C}$ per $24 \mathrm{~h}$, questo terreno è stato utilizzato sia per la ricerca dei coliformi che per quella di $E$. coli, basandosi sulla reazione cromogena delle colonie.

Prima e dopo i trattamenti e ai diversi intervalli di conservazione sono stati misurati $\mathrm{pH}$ (pH-metro Knick Portamass ${ }^{\circledR}$ ), temperatura (Checktemp ${ }^{\circ} \mathrm{C}$; Hanna Instruments,
Woonsocket, RI, USA) e indice rifrattometrico ( ${ }^{\circ}$ Brix; rifrattometro Atago ${ }^{\circledR}$ ).

La valutazione dell'effetto della sonicazione sulle caratteristiche organolettiche del succo è stata condotta attraverso un'analisi sensoriale di tipo descrittivo da parte di sei giudici precedentemente addestrati. A tal proposito i descrittori considerati si riferivano alle caratteristiche dell'aspetto (torbidità, presenza di particelle in sospensione, intensità di colore), dell'aroma (fruttato di mela, erbaceo, dolce, bruciato, cotto, metallico, acido, muffa) e del gusto (fruttato di mela, erbaceo, amaro, dolce, bruciato, cotto, metallico, acido, muffa, astringenza, consistenza della polpa) e a un giudizio di accettabilità generale. La valutazione è stata effettuata secondo una scala da 1 (assente, sgradevole, ecc.) a 9 (molto gradevole, molto intenso, ecc.). I panel test sono stati effettuati in concomitanza delle analisi microbiologiche.

I dati ottenuti dall'analisi sensoriale sono stati sottoposti ad analisi della varianza (ANOVA) utilizzando il pacchetto statistico PASW Statistics 18 (2009). La distribuzione dei dati è stata valutata con il test di Kolmogorov-Simimov. Il test post hoc è stato effettuato con il test HSD di Tukey la cui differenza tra le medie è significativa al livello 0,05 . L'analisi ha valutato l'effetto del trattamento, del tempo di conservazione e la relativa interazione (Sampedro et al., 2009; Campo et al., 2005; Dello Staffolo et al., 2004; Esti et al., 2002).

\section{Risultati}

I valori di temperatura rilevati pre- e postsonicazione erano rispettivamente di $11.8 \pm 0.8$ e $37.6 \pm 1.1^{\circ} \mathrm{C}$. Il riscaldamento del succo è stato effettuato in bagno termostatato ripetendo la stessa cinetica di salita della temperatura verificatasi durante il trattamento di sonicazione. I valori di $\mathrm{pH}$ e dell'indice zuccherino, rilevati pre- e post trattamento e nei tempi successivi, non hanno evidenziato variazioni nelle diverse tesi e nel tempo presentandosi rispettivamente intorno ai $3.5 \pm 0.1$ e $11.5 \pm 0.2{ }^{\circ}$ Brix per tutti i campioni.

Le Figure 1 e 2 rispettivamente mostrano gli andamenti della carica microbica totale mesofila (CMT) e psicrofila (CPT) e dei lieviti e muffe rilevate per le tre tesi $(\mathrm{S}, \mathrm{T}, \mathrm{C})$ nei quattro tempi. Dal confronto delle curve dei mesofili è possibile osservare che al T0 il succo di controllo presenta valori leggermente più alti rispetto al succo sonicato e al riscaldato. L'andamento delle curve di crescita ha presentato una progressione simile nel campione riscaldato e nel controllo, mentre si evidenzia un progressivo e continuo decremento nel succo sonicato fino al T21. La CMT del succo sonicato presenta una riduzione di circa 3 log
UFC/mL. Osservando le curve relative alla CPT per le tre tesi si può notare l'andamento nettamente differente dei due succhi trattati rispetto al controllo. Al T0 i valori della CPT per il succo di controllo e il riscaldato sono quasi sovrapponibili, ma nel tempo seguono andamenti diversi, infatti la curva del controllo inizia la sua ascesa al T7 che continua fino al T14 mentre quella del riscaldato decresce al T7 per poi aumentare al T14 non raggiungendo, tuttavia, i valori del controllo. La CPT del succo sonicato al $21^{\circ}$ giorno presenta circa 5 log UFC/mL in meno rispetto al controllo. Dal confronto dello stesso parametro tra il succo riscaldato e il controllo si deduce che l'effetto del calore determina una riduzione della carica batterica di circa $1 \log$ UFC/mL al T14 che diventa minima al T21. Risulta quindi scorporabile la valutazione dell'effetto termico rispetto a quello degli ultrasuoni che evidenziano una spiccata azione battericida/batteriostatica anche sul lungo periodo. Per quanto riguarda le muffe, nel tempo si assiste ad una riduzione della crescita che per il succo controllo al T14 è quasi nulla a differenza dei succhi trattati (Figura 2). Confrontando le curve relative alla crescita dei lieviti si può osservare un andamento simile nel tempo per le tre tesi in tutti gli intervalli di campionamento, tuttavia il succo sonicato presenta valori costantemente più bassi di circa 1 log UFC/mL. La stima dei lattobacilli a 22 e a $30^{\circ} \mathrm{C}$, dei coliformi a $37^{\circ} \mathrm{C}$ e di $E$. coli è stata inferiore a $1 \mathrm{UFC} / \mathrm{mL}$.

Per quanto riguarda i risultati relativi all'analisi sensoriale, sono riportati solo i descrittori risultati statisticamente significativi in relazione ai due parametri tempo (Tabella 1) e trattamento (Tabella 2). I risultati relativi al parametro tempo si riferiscono ai 21 giorni per la valutazione dei descrittori aspetto e odore e ai 14 giorni per quello riguardante l'aroma, poiché i giudici hanno scelto di non assaggiare i campioni al $21^{\circ}$ giorno. Per quanto riguarda l'accettabilità generale, considerando il parametro tempo, il valore più alto si è osservato in corrispondenza del T7 con un valore medio di 6,6 rispetto al valore medio più basso 3,7 rilevato in corrispondenza del T21. Per quanto riguarda l'aspetto i caratteri: intensità di colore, torbidità e presenza di particelle in sospensione presentano valori più alti al $\mathrm{T} 0$. In relazione all'odore il carattere maggiormente percepito è quello di muffa in corrispondenza del $21^{\circ}$ giorno. Dal confronto dei trattamenti, il succo sonicato presenta il valore medio di accettabilità generale più alto, pari a 6,5, seguono il riscaldato e il controllo con un valore medio rispettivamente di 5,2 e 4,8. Inoltre il succo sonicato presenta i valori più bassi di intensità di colore pari a 5,5, di torbidità, $3,7 \mathrm{e}$ di presenza di particelle in sospensione 2,6. Per quanto riguarda l'odore il succo sonicato ha conservato il carattere erbaceo con un valore medio di 4,7 contro il 3,4 del riscaldato e il 3,6 
del controllo e presenta il valore medio di muffa più basso, 1,3 rispetto al riscaldato e al controllo i cui valori medi sono rispettivamente pari a 1,8 e 2,2 .

\section{Discussione}

Obiettivo dello studio era acquisire informazioni riguardanti il profilo microbiologico e sensoriale di un succo di mela sottoposto ad un trattamento di sonicazione da applicare in alternativa ai tradizionali sistemi che prevedono l'utilizzo del calore. Data la ridotta presenza di informazioni in letteratura, in questa prima indagine non si è voluto effettuare un confronto con un canonico trattamento di pastorizzazione ma si è preferito comprendere se l'efficacia dell'applicazione degli US sia dovuta ai fenomeni fisici di cavitazione 0 al rialzo termico che, anche se contenuto, comunque si manifesta. Per tale motivo il confronto è stato fatto con un succo sottoposto ad una temperatura di circa $37^{\circ} \mathrm{C}$.

La scelta di un'applicazione di ultrasuoni caratterizzata da ampiezza dell'onda (A) pari al 100\%, durata di sonicazione (D) $300 \mathrm{~s} / 100$ $\mathrm{mL}$ e pulsazione $(\mathrm{P})$ pari a 1 è stata dettata da esperienze precedenti condotte su latte crudo (Montemurro et al., 2011) in cui questi rappresentavano i parametri di trattamento più intensi. Il latte è un alimento più complesso rispetto al succo di frutta, quindi non è possibile effettuare un confronto tra le due matrici, tuttavia dal punto di vista sensoriale, il latte sonicato con il medesimo trattamento del presente lavoro è risultato nel complesso sgradevole. I dati ottenuti seppur preliminari sono incoraggianti.

L'andamento delle curve relative alla CPT presenta un comportamento che denota l'assenza di cellule stressate nel succo controllo
Tabella 1. Valutazione sensoriale in relazione al tempo di osservazione.

\begin{tabular}{|c|c|c|c|c|c|}
\hline \multirow[t]{2}{*}{ Descrittore } & \multicolumn{4}{|c|}{ Tempo (giorni) } & \multirow[t]{2}{*}{ ESM } \\
\hline & T0 & $\mathrm{T} 7$ & T14 & T21 & \\
\hline \multicolumn{6}{|l|}{ Aspetto } \\
\hline Intensità di colore & $7.0^{\mathrm{b}}$ & $6.5^{\mathrm{ab}}$ & $5.6^{\mathrm{ab}}$ & $5.5^{\mathrm{a}}$ & $0.4^{*}$ \\
\hline Torbidità & $6.6^{\mathrm{a}}$ & $5.5^{\mathrm{ab}}$ & $5.1^{\mathrm{a}}$ & $5.2^{\mathrm{ab}}$ & $0.4^{*}$ \\
\hline Presenza di particelle in sospensione & $4.7^{\mathrm{b}}$ & $2.7^{\mathrm{a}}$ & $3.1^{\mathrm{ab}}$ & $3.2^{\mathrm{ab}}$ & $0.4^{* *}$ \\
\hline \multicolumn{6}{|l|}{ Odore } \\
\hline Fruttato di mela & $4.8^{c}$ & $4.9^{\mathrm{d}}$ & $3.9^{\mathrm{b}}$ & $3.2^{\mathrm{a}}$ & $0.4^{*}$ \\
\hline Erbaceo & $4.7^{\mathrm{b}}$ & $4.3^{\mathrm{ab}}$ & $3.8^{\mathrm{ab}}$ & $2.8^{\mathrm{a}}$ & $0.4^{*}$ \\
\hline Dolce & $4.7^{\mathrm{b}}$ & $3.9^{\mathrm{ab}}$ & $3.2^{\mathrm{ab}}$ & $2.9^{\mathrm{a}}$ & $0.4^{*}$ \\
\hline Muffa & $1.2^{\mathrm{a}}$ & $1.2^{\mathrm{a}}$ & $2.1^{\mathrm{ab}}$ & $2.4^{\mathrm{b}}$ & $0.3^{* * *}$ \\
\hline \multicolumn{6}{|l|}{ Aroma } \\
\hline Muffa & 1.0 & 1.1 & 1.5 & - & 0.1 (ns) \\
\hline Polpa & $4^{\mathrm{ab}}$ & $5.0^{\mathrm{b}}$ & $1.5^{\mathrm{a}}$ & - & $0.7^{*}$ \\
\hline Accettabilità generale & $6.4^{\mathrm{ab}}$ & $6.6^{\mathrm{b}}$ & $5.2^{\mathrm{ab}}$ & $3.7^{\mathrm{a}}$ & $0.4^{* * *}$ \\
\hline
\end{tabular}

ESM, errore standard medio; ns, non significativo. Descrittore 1 (assente, sgradevole, ecc.) - 9 (molto gradevole, molto intenso, ecc.). ${ }^{*} \mathrm{P}<0,05 ; * * \mathrm{P}<0,01 ; * * \mathrm{P}<0,001$.

Tabella 2. Valutazione sensoriale in relazione ai trattamenti.

\begin{tabular}{lcccc} 
Descrittore & \multicolumn{3}{c}{ Trattamento } & ESM \\
& $\mathrm{C}$ & $\mathrm{S}$ & $\mathrm{T}$ & \\
& & & & \\
Aspetto & 6.5 & 5.5 & 6.4 & $0.3(\mathrm{~ns})$ \\
$\quad$ Intensità di colore & $7.2^{\mathrm{c}}$ & $3.7^{\mathrm{a}}$ & $5.8^{\mathrm{b}}$ & $0.3^{* * *}$ \\
Torbidità & & $2.6^{\mathrm{a}}$ & $2.8^{\mathrm{ab}}$ & $0.3^{* * *}$ \\
$\quad$ Presenza di particelle in sospensione & $4.9^{\mathrm{b}}$ & & \\
Odore & & & & \\
Fruttato di mela & 3.9 & 4.9 & 3.8 & $0.4(\mathrm{~ns})$ \\
Erbaceo & $3.6^{\mathrm{ab}}$ & $4.7^{\mathrm{b}}$ & $3.4^{\mathrm{a}}$ & $0.4^{*}$ \\
Dolce & 3.8 & $3.9^{\mathrm{a}}$ & 3.5 & $0.4(\mathrm{~ns})$ \\
Muffa & $2.2 \mathrm{~b}$ & $1.3^{\mathrm{a}}$ & $1.8^{\mathrm{ab}}$ & $0.2^{*}$ \\
\hline Aroma & & & & \\
$\quad$ Muffa & $1.0^{\mathrm{a}}$ & $1.0^{\mathrm{b}}$ & $1.4^{\mathrm{c}}$ & $0.1^{*}$ \\
Polpa & 5.5 & 2.8 & 3.7 & $0.6^{\dagger}$ \\
Accettabilità generale & $4.8^{\mathrm{b}}$ & $6.5^{\mathrm{a}}$ & $5.2^{\mathrm{ab}}$ & $0.3^{* *}$ \\
\hline
\end{tabular}

ESM, errore standard medio; C, controllo; S, ultrasuoni; T, riscaldato; ns, non significativo. Descrittore 1 (assente, sgradevole, ecc.) - 9 (molto gradevole, molto intenso, ecc.). $\dagger \mathrm{P}<0,10 ; * \mathrm{P}<0,05 ; * * \mathrm{P}<0,01 ; * * * \mathrm{P}<0,001$.

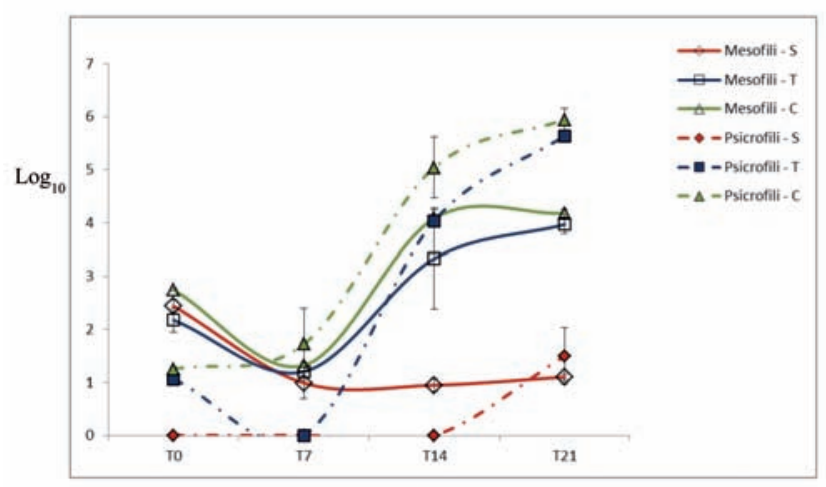

Figura 1. Andamento delle cariche microbiche mesofile e psicrofile (media e deviazione standard).

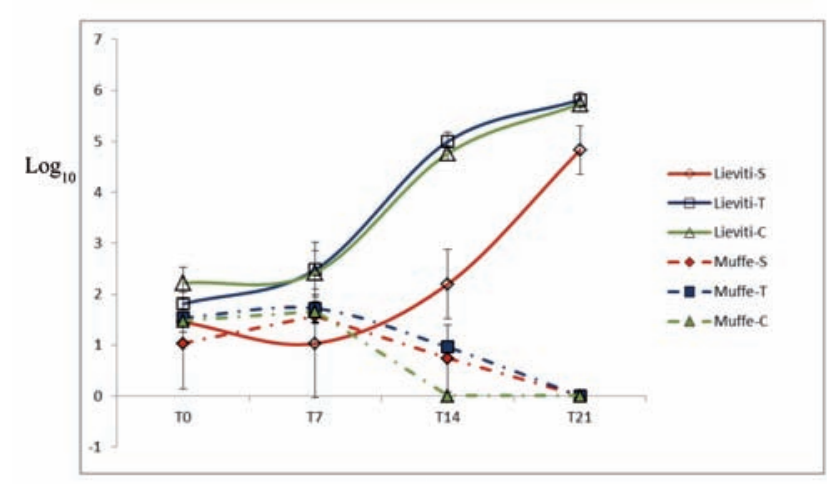

Figura 2. Andamento dei lieviti e delle muffe (media e deviazione standard). 
rispetto al riscaldato e al sonicato. La CMT, invece, presenta un periodo di adattamento alla temperatura di refrigerazione, dal T0 al T7, nelle tre tesi. I lieviti hanno mostrato, in assoluto, la maggiore resistenza al trattamento con gli ultrasuoni, dato che conferma i risultati riportati da Gabriel Alonzo (2012) in uno studio in cui valutava l'azione degli ultrasuoni su lieviti e altri microrganismi inoculati in succo di mela. In merito alla minore efficacia sui lieviti, in letteratura sono quasi assenti le informazioni e i dati riportati riguardano i prodotti fermentati. Infatti Chisti (2003) e Matsuura et al. (1994) affermano che gli ultrasuoni favoriscono la crescita dei lieviti diminuendo la concentrazione, quindi l'azione inibente, dell'anidride carbonica nel sidro di mele. Bujons et al. (1988) segnalano invece una maggiore sintesi di steroli nel lievito di birra trattato con US. Non sono state evidenziate cariche rilevanti di coliformi ed $E$. coli nel controllo e nei trattati già al T0, questo indica bassa contaminazione iniziale della frutta utilizzata per la produzione del succo, risultato che conferma quanto riportato da Suarez-Jacobo et al. (2010). Tuttavia non va dimenticato che la US Food and Drug Administration (2001), in seguito a casi di malattia legati all'ingestione di succo di mela contaminato con E. coli $0157: \mathrm{H} 7$ ha emesso delle linee guida con uno standard di riduzione che impone un abbattimento minimo di $5 \log$ della carica dei patogeni nel succo pronto al consumo (USFDA, 2001).

L'analisi organolettica non si è prefissa lo scopo di evidenziare un carattere che meglio identifica il succo ma ha inteso valutare in via esplorativa le caratteristiche del prodotto sonicato. Il giudizio generale espresso dai giudici, attraverso il panel test, è stato a favore del succo sonicato, seguono il riscaldato e il controllo. Non è stato semplice valutare il colore dei campioni analizzati. Al fine di rispettare le modalità di produzione del succo opalescente che, grazie alla presenza di particelle solide di piccolissime dimensioni, quali frammenti di cellule vegetali, pectine e proteine (Kilara e Van Buren, 1989) risulta organoletticamente più simile al succo fresco con un corpo che lo rende più gradevole al consumatore, si è deciso di non filtrare i campioni. Questo ha reso più difficile la determinazione del colore che $s i$ è basata solo sulla valutazione dell'intensità mostrata dai nostri campioni rispetto a quella di un succo standard presente in commercio.

La comprensione dell'effetto degli US sull'aspetto e le caratteristiche chimico-fisiche del prodotto necessita di ulteriori approfondimenti, in quanto il processo di trasformazione delle mele richiede l'inattivazione degli enzimi pectinolitici (pectinmetilesterasi) al fine di mantenere la naturale opalescenza del succo. Per far ciò il succo all'uscita dalla pressa subisce un trattamento termico (high temperature short time) con conseguente imbrunimento a seguito sia dell'ossidazione dei composti fenolici che della reazione di Maillard. Gli ultrasuoni potrebbero essere impiegati non solo come trattamento alternativo alla pastorizzazione ma anche con la finalità di mantenere la naturale opalescenza del succo di mela inattivando gli enzimi pectinolitici ed evitandone l'imbrunimento derivante dall'azione della fenolasi (polifenolossidasi) sulle sostanze polifenoliche contenute nelle mele.

\section{Conclusioni}

Concludendo possiamo affermare che il trattamento con US, testato in questa esperienza, ha fornito risultati interessanti per quanto riguarda l'abbattimento delle cariche microbiche. In generale, l'utilizzo degli ultrasuoni sembra essere una buona alternativa alla pastorizzazione per la produzione di un succo di mela di buona qualità. Tuttavia, sarà necessario confermare i dati ottenuti con altre prove di shelf life, aumentando il tempo di osservazione per le analisi microbiologiche e sensoriali.

Al fine di ottenere maggiori informazioni sulle caratteristiche organolettiche e nutritive di un prodotto ottenuto con mild technology, alle precedenti analisi si assoceranno quelle chimico-fisiche.

\section{Bibliografia}

Adekunte A, Tiwari BK, Scannell A, Cullen PJ, O'Donnell C, 2010. Modelling of yeast inactivation in sonicated tomato juice. Int $\mathrm{J}$ Food Microbiol 137:116-20.

Ayhan Z, Zhang QH, Min DB, 2002. Effects of pulsed electric field processing and storage on the quality and stability of single strength orange juice. J Food Protect 65:1623-7.

Bermúdez-Aguirre D, Corradini MG, Mawson R, Barbosa-Cánovas GV, 2009. Modeling the inactivation of Listeria innocua in raw whole milk treated under thermo-sonication. Innov Food Sci Emerg 10:172-8.

Bignon J, 1996. Cold pasteurizers Hyperbar for the stabilization of fresh fruit juices. Fruit Process 6:46-8.

Bujons J, Guajardo R, Kyler KS, 1988. Enantioselective enzymatic sterol synthesis by ultrasonically stimulated baker's yeast. J Am Chem Soc 110:604-6.

Bump VL, 1989. Apple pressing and juice extraction. In: Downing DL, ed. Processed apple products. Van Nostrand Reinhold, New York, NY, pp 53-82.

Campo E, Ferreira V, Escudero A, Cacho J, 2005. Prediction of the wine sensory prop- erties related to grape variety from dynamic-headspace gas chromatography-olfactometry data. J Agr Food Chem 53:5682-90.

Chisti Y, 2003. Sonobioreactors: using ultrasound for enhanced microbial productivity. Trends Biotechnol 21:89-93.

Dalpozzo D, 2010. Focus food: i succhi di frutta cercano massima naturalità, nuovi gusti $\mathrm{e}$ pack leggero. Un mercato in cui il consumatore cerca convenienza ma non accetta compromessi sulla qualità. MARK UP 187 mensile de Il Sole 240re. Disponibile al sito: http://www.mark-up.it/articoli/0,1254,41_ART_4296,00.html

D’Amico DJ, Silk TM, Wu J, Guo M, 2006. Inactivation of microorganism in milk and apple cider treated with ultrasound. J Food Protect 69:556-63.

Dello Staffolo M, Bertola N, Martino M, Bevilacqua A, 2004. Influence of dietary fiber addition on sensory and rheological properties of yogurt. Int Dairy J 14:263-8.

Donahue DW, Canitez N, Bushway AA, 2004. UV inactivation of E. coli 0157:H7 in apple juice: quality sensory and shelf life analysis. J Food Process Pres 28:368-87.

Esti M, Cinquanta L, Sinesio F, Moneta E, Di Matteo M, 2002. Physicochemical and sensory fruit characteristics of two sweet cherry cultivars after cool storage. Food Chem 76:399-405.

Evrendilek GA, Zhang QH, Richter ER, 1999. Inactivation of Escherichia coli 0157:H7, Escherichia coli 8739 in apple juice by pulsed electric fields. J Food Protect 62:793-6.

Franz CMAP, Specht I, Cho G, Graef V, Stahl MR, 2009. UV-C-inactivation of microorganisms in naturally cloudy apple juice using novel inactivation equipment based on Dean vortex technology. Food Control 20:1103-7.

Gabriel Alonzo A, 2012. Microbial inactivation in cloudy apple juice by multi-frequency Dynashock power ultrasound. Ultrason Sonochem 19:346-51.

Guerrero-Beltrán JA, Barbosa-Cánovas GV, 2005. Reduction of Saccharomyces cerevisiae, Escherichia coli and Listeria innocua in apple juice by ultraviolet light. J Food Process Eng 28:437-52.

Kelly AL, Zeece M, 2009. Applications of novel technologies in processing of functional foods. Aust J Dairy Technol 64:12-5.

Kilara A, Van Buren JP, 1989. Clarification of apple Juice. In: Downing DL, ed. Processed apple products. Van Nostrand Reinhold, New York, NY, pp 83-96.

Lee KW, Kim YJ, Kim D0, Lee HJ, Lee CY, 2003. Major phenolics in apples and their contribution to total antioxidant capacity. J Agr Food Chem 51:6516-20.

Leontowicz M, Gorinstein S, Leontowicz H, Krzeminski R, Lojek A, Katrich E, Cizm M, 
Martin-Bellooso 0, Soliva-Fortuny R, Piyasena P, Mohareb E, McKellar RC, 2003. Haruenkit R, Trakhtenberg S, 2003. Apple and pear peel and pulp and their influence on plasma lipids and antioxidant potentials in rats fed cholesterol-containing dietes. J Agr Food Chem 51:5780-5.

Matsuura K, Hirotsune M, Nunokawa Y, Satoh M, Honda K, 1994. Acceleration of cell growth and ester formation by ultrasonic wave irradiation. J Ferment Bioeng 77:3640.

Montemurro F, Marchesini G, Balzan S, Fasolato L, Segato S, Andrighetto I, Novelli E, 2011. Utilizzo degli ultrasuoni nella debatterizzazione del latte: risultati preliminari. Scienza e Tecnica LattieroCasearia 62:327-31.

Patil S, Bourke P, Kelly B, Frías J, Cullen PJ, 2009. The effects of acid adaptation on Escherichia coli inactivation using power ultrasound. Innov Food Sci Emerg 10:48690.
Inactivation of microbes using ultrasound: a review. Int J Food Microbiol 87:207-16.

Pompei C, 2005. La trasformazione industriale di frutta ed ortaggi. Tecnologie per la produzione di conserve e semiconserve vegetali. Edagricole-Edizioni Agricole de Il Sole 24 ORE, Milano, Italia.

Salleh-Mack SZ, Roberts JS, 2007. Ultrasound pasteurization: the effects of temperature soluble solids organic acids and $\mathrm{pH}$ on the inactivation of Escherichia coli ATCC 25922. Ultrason Sonochem 14:323-9.

Sampedro F, Geveke DJ, Fan X, Rodrigo D, Zhang QH, 2009. Shelf-life study of an orange juice-milk based beverage after PEF and thermal processing. J Food Sci $74: 2$.

Suárez-Jacobo A, Gervilla R, Guamis B, RoigSagués AX, Saldo J, 2010. Effect of UHPH on indigenous microbiota of apple juice. A preliminary study of microbial shelf-life.
Int J Food Microbiol 136:261-7.

USFDA, 2001. Hazard analysis and critical control point (HACCP); procedures for the safe and sanitary processing and importing of juice; final rule. Disponibile al sito: http://www.fda.gov/ohrms/dockets/98fr/011 901b.htm

Villamiel M, de Jong P, 2000. Influence of highintensity ultrasound and heat treatments in continuous flow on fat, proteins, and native enzymes of milk. J Agr Food Chem 48:472-8.

Vojdani J, Beuchat L, Tauxe R, 2008. Juiceassociated outbreaks of human illness in the United States, 1995 through 2005. J Food Protect 71:356-64.

Wright JR, Sumner SS, Hackney CR, Pierson MD, Zoecklein BW, 2000. Efficacy of ultraviolet light for reducing Escherichia coli 0157:H7 in unpasteurized apple cider. J Food Protect 63:563-7. 